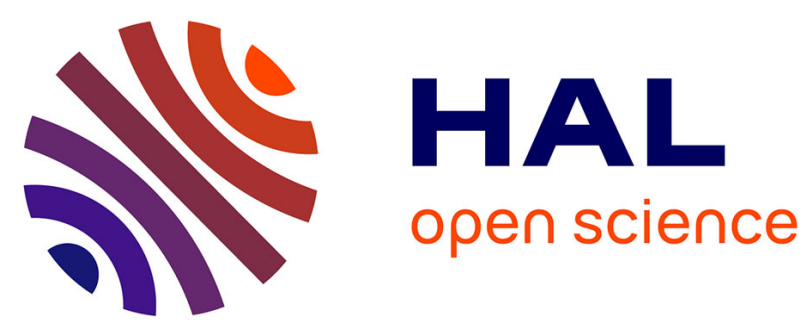

\title{
Minimal time problem for a fed-batch bioreactor with a non admissible singular arc
}

\author{
Térence Bayen, Francis Mairet, Marc Mazade
}

\section{To cite this version:}

Térence Bayen, Francis Mairet, Marc Mazade. Minimal time problem for a fed-batch bioreactor with a non admissible singular arc. European Control Conference (ECC), Université de Strasbourg (Unistra). Strasbourg, FRA. IEEE Control Systems Society., Jun 2014, Strasbourg, France. pp.165170, 10.1109/ECC.2014.6862444 . hal-01095694

\section{HAL Id: hal-01095694 \\ https://inria.hal.science/hal-01095694}

Submitted on 16 Dec 2014

HAL is a multi-disciplinary open access archive for the deposit and dissemination of scientific research documents, whether they are published or not. The documents may come from teaching and research institutions in France or abroad, or from public or private research centers.
L'archive ouverte pluridisciplinaire HAL, est destinée au dépôt et à la diffusion de documents scientifiques de niveau recherche, publiés ou non, émanant des établissements d'enseignement et de recherche français ou étrangers, des laboratoires publics ou privés. 


\title{
Minimal time problem for a fed-batch bioreactor with a non admissible singular arc
}

\author{
Terence Bayen ${ }^{1,2}$ and Francis Mairet ${ }^{3}$ and Marc Mazade ${ }^{1}$
}

\begin{abstract}
In this paper, we consider an optimal control problem for a system describing a fed-batch bioreactor with one species and one substrate. Our aim is to find an optimal feedback control in order to steer the system to a given target in minimal time. The growth function is of Haldane type implying the existence of a singular arc. Unlike many studies on the minimal time problem governed by an affine system w.r.t. the control with one input, we assume that the singular arc is non-necessary controllable. This brings interesting issues in terms of optimal synthesis. Thanks to the Pontryagin Maximum Principle, we provide the optimal synthesis of the problem, It turns out that singular extremal trajectories are no longer optimal on a subset of the singular arc.
\end{abstract}

\section{INTRODUCTION}

The study of the minimal time control problem for affine systems with one input:

$$
\dot{x}=f(x)+u g(x), \quad x \in \mathbb{R}^{n}, \quad|u| \leq 1,
$$

has been investigated a lot in the literature, see [6] for $n=2$ and references herein. One often encounters singular trajectories which appear when the switching function of the system is vanishing on a sub-interval. In order to find an issue to an optimal control problem governed by (1), studies often require that the singular arc is controllable which means that the singular control $u_{s}$ allowing the trajectory to stay on the singular arc is supposed to verify the inequality:

$$
\left|u_{s}\right| \leq 1 \text {. }
$$

However, one cannot in general show that this assumption holds. In fact, if the singular arc is of first order, the expression of the singular control $u_{s}$ is in general complicated as it depends both on the state and the adjoint state. The objective of this work is to study a minimal time control problem in the plane, that is $n=2$ where the singular control is not always admissible i.e. (2) is not always satisfied.

We consider a fed-batch bioreactor with one species and one substrate. Our aim is to find an optimal feedback control that steers the system in minimal time to a given target where the substrate concentration is less than a prescribed value, see [11]. Finding an optimal feeding strategy can significantly increase the performance of the system and has

\footnotetext{
${ }^{1}$ Université Montpellier 2, Case courrier 051, 34095 Montpellier cedex 5, France. tbayen@math.univ-montp2.fr, mmazade@math.univ-montp2.fr

'2INRA-INRIA 'MODEMIC' team, INRIA Sophia-Antipolis Méditerranée, UMR INRA-SupAgro 729 'MISTEA' 2 place Viala 34060 Montpellier.

$\begin{array}{rlrrr}3 & \text { Inria 'BIOCORE' } & \text { team, } & \text { Sophia-Antipolis, } 2004 \text { route } \\ \text { des } & \text { Lucioles, } 06902 & \text { Sophia } & \text { Antipolis Cedex, France }\end{array}$ francis.maireteinria.fr
}

several advantages from a practical point of view (see e.g. [1], [2], [7], [8], [9], [11]).

Whenever the growth function is of Monod type, then one can prove that the optimal feeding strategy is bang-bang [11]. This means that the reactor is filled until its maximum volume with the maximum input flow rate. Then, microorganisms consume the substrate until reaching a reference value. In the case where the growth function is of Haldane type (in case of substrate inhibition), this strategy is not optimal. In fact, one can prove (see [11]) that the optimal strategy is singular. It consists in reaching a substrate concentration $\bar{s}$ (corresponding to the maximum of the growth rate function) in minimal time. Then, the substrate concentration is kept constant to this value until reaching the maximal volume. This can be proved by using either the Pontryagin Maximum Principle (PMP) or the clock form [5], [10].

In the present work, we are interested in studying the optimal synthesis for Haldane-type growth function whenever the singular arc is no longer admissible from a certain volume value. This happens when the singular control is larger than the maximal input flow rate which is allowed in the system. It follows that there exists a volume value above which singular extremal trajectories are no longer optimal. Whereas in [11], [2], [1], we suppose that the maximal volume value can be reached by the singular arc, there exists a volume value above which it is not possible to keep the substrate concentration equal to $\bar{s}$ in the system. The main issue of the paper is the following. We aim at determining an optimal feedback control whenever the the singular arc becomes non admissible (i.e. the singular control saturates one of the two bounds on the control).

First, one cannot apply the clock form [5] (that allows a direct comparison between the cost of two trajectories) as we do not have a natural candidate for optimality in this case. In particular, optimal strategies developed in [2], [1] are no longer admissible. Thanks to Pontryagin's principle, we show that the optimal synthesis of the problem is rather different as in [11]. We introduce solutions of the system backward in time with the maximum control that allow to determine where the switching time occurs for optimal trajectories. Our main result is that a singular extremal trajectory stops to be optimal before reaching the maximal admissible value (this is rather non-intuitive and is slightly different as the controllable case). We show that there exists a maximal volume value above which a singular trajectory is not optimal. We also provide numerical switching curves for the control which allow to determine an optimal feedback control of the problem. 
The paper is organized as follows. The first section states the optimal control problem. We also recall the optimality result of [11] and we apply the PMP. The second section is devoted to the optimal synthesis of the problem when the singular is not admissible everywhere. We first describe the curves that are solution of the system backward in time and that allow to determine properties of optimal trajectories. Then, we state our main results in the case where the singular arc is never controllable (Proposition 2) and whenever it is controllable until a certain volume (Proposition 3). The last section is devoted to numerical simulations. We exhibit a numerical switching curve for the study that allows to provide an optimal feedback control of the problem.

\section{General RESUlts}

In this section, we state the optimal control problem, we make a review of the standard optimality result in [11] when the singular arc is controllable, and we apply the Pontryagin Maximum Principle (see [12]) that will be used in the next section.

\section{A. Statement of the problem}

We consider a system describing a fed-batch bioreactor with one species and one substrate:

$$
\left\{\begin{array}{l}
\dot{x}=x\left(\mu(s)-\frac{u}{v}\right) \\
\dot{s}=-\mu(s) x+\frac{u}{v}\left(s_{i n}-s\right), \\
\dot{v}=u
\end{array}\right.
$$

where $x$ represents the concentration of micro-organisms, $s$ the concentration of substrate, and $v$ is the volume of the tank. The input substrate concentration is denoted by $s_{\text {in }}>$ 0 , and $u$ is the input flow rate in the system. For convenience, we have taken yield coefficient equal to one (by rescaling the equation). The function $s \longmapsto \mu(s)$ is the growth function of Monod or Haldane type (see [13]). In the following, we consider that $u$ takes values within the set:

$$
\mathcal{U}:=\left\{u:[0,+\infty) \rightarrow\left[0, u_{\max }\right] \mid u \text { meas. }\right\} .
$$

Here $u_{\max }$ denotes the maximum input flow rate in the system. In the following, we can take by time scaling $u_{\max }=1$. The target we consider is defined by:

$$
\mathcal{T}=\mathbb{R}_{+}^{*} \times\left[0, s_{\text {ref }}\right] \times\left\{v_{m}\right\} .
$$

For $u \in \mathcal{U}$, let $t_{\xi_{0}}(u)$ the time to steer (3) from an initial condition $\xi_{0}:=\left(x_{0}, s_{0}, v_{0}\right) \in \mathbb{R}_{+}^{*} \times\left[0, s_{i n}\right] \times\left[0, v_{m}\right]$. The optimal control problem becomes:

$$
\inf _{u \in \mathcal{U}} t_{\xi_{0}}(u) \text {, s.t. } \xi(t(u)) \in \mathcal{T},
$$

where $\xi(\cdot)$ denotes the unique solution of (3) for the control $u$ that starts at $\xi_{0}$. One essential feature in the system (3) is that the quantity

$$
M:=v\left(x+s-s_{i n}\right),
$$

is conserved along any trajectory of (3), hence $M$ is constant and equal to $v_{0}\left(x_{0}+s_{0}-s_{i n}\right)$. From (7), we obtain:

$$
x=\frac{M}{v}+s_{i n}-s,
$$

and system (3) can be put into a two-dimensional system:

$$
\left\{\begin{array}{l}
\dot{s}=-\mu(s)\left(\frac{M}{v}+s_{i n}-s\right)+\frac{u}{v}\left(s_{i n}-s\right), \\
\dot{v}=u
\end{array}\right.
$$

On can easily show that the set $\left[0, s_{i n}\right] \times \mathbb{R}_{+}^{*}$ is invariant by (9). Notice that if we define $x$ by (8), the micro-organisms concentration may not be positive. This may happen when $M \leq 0$ which means that initial conditions of microorganisms and substrate are low. Therefore, we consider initial conditions for (9) in the domain $\mathcal{D}$ defined by:

$$
\mathcal{D}:=\left\{(s, v) \in\left[0, s_{i n}\right] \times\left(0, v_{m}\right] \mid \frac{M}{v}+s_{\text {in }}-s>0\right\} .
$$

In the rest of the paper, we write $u(\cdot)$ a control in open loop and $u[\cdot]$ a feedback control depending on the state $(s, v)$.

\section{B. Optimal synthesis when the singular arc is admissible}

In this part, we review the result of [11] on optimal trajectories for problem (6) that will allow to introduce the problem without supposing that the singular arc is admissible.

Next, we say that the growth function $\mu$ is of Monod type if $\mu(s)=\frac{\bar{\mu} s}{k+s}$ with $\bar{\mu}>0$ and $k>0$.

Theorem 1: Assume that $\mu$ is of Monod type. Then, the optimal feedback control $u_{M}$ steering any initial condition in $\mathcal{D}$ to the target $\mathcal{T}$ is:

$$
u_{M}[s, v]:=\left\{\begin{array}{l}
1 \text { if } v<v_{m} \\
0 \text { if } v=v_{m}
\end{array}\right.
$$

In other words, the optimal strategy is fill and wait, and it consists in filling the tank with maximum input flow rate until $v=v_{m}$, and then we let $u=0$ until $s$ reaches the value $s_{\text {ref }}$ (if necessary).

In the rest of the paper, we will consider only the case where the growth function $\mu$ is of Haldane type i.e.

$$
\mu(s)=\frac{\bar{\mu} s}{g s^{2}+s+k},
$$

with $\bar{\mu}>0, g>0$, and $k>0$. In this case, $\mu$ has exactly one maximum over $\mathbb{R}_{+}$, that we denote $\bar{s}$, and we suppose that $\bar{s}>s_{\text {ref }}$ (which means that the reference concentration to achieve is sufficiently small). The optimal synthesis in this case is rather different as for Monod growth function.

Theorem 2: Assume that $\mu$ is of Haldane type and that the following assumption holds:

$$
\mu(\bar{s})\left[\frac{M}{s_{i n}-\bar{s}}+v_{m}\right] \leq 1 .
$$

Then, the optimal feedback control $u_{H}$ to reach the target is given by

$$
u_{H}[s, v]:=\left\{\begin{array}{rlll}
0 & \text { if } v=v_{m} & \text { or } \quad s>\bar{s} \\
1 & \text { if } s<\bar{s} & \text { and } & v<v_{m} \\
u_{s}(v) & \text { if } s=\bar{s} & \text { and } & v<v_{m}
\end{array}\right.
$$


where

$$
u_{s}(v):=\mu(\bar{s})\left[\frac{M}{s_{i n}-\bar{s}}+v\right] .
$$

This result can be proved via the clock form, see e.g. [11]. This tool is based on Green's Theorem and allows a direct comparison of the cost between two trajectories steering an initial point to the same target point.

Here we have emphasized the assumption (12) ensuring the system to stay on the singular arc (see e.g. [7], [1]). The control $u_{s}$ is singular (see section II-C). It allows to maintain the substrate concentration equal to $\bar{s}$. It can be written $u_{s}(v)=\frac{\mu(\bar{s}) x}{v\left(s_{i n}-\bar{s}\right)}$ so that $u_{s} \geq 0$. Therefore, assumption (12) is essential to ensure that $u_{s}(v)$ satisfies the upper bound $u_{s}(v) \leq 1$ for all $v \leq v_{m}$.

The objective of this paper is to provide an optimal synthesis of the problem whenever (12) is non-necessarily satisfied. Note that in practice, one should start the fed-batch with a high biomass concentration (i.e. high $M$ ) in order to speed up the process, so that condition (12) can no longer be satisfied.

\section{Pontryagin maximum principle}

In this part we apply Pontryagin maximum principle on (6). Let $H:=H\left(s, v, \lambda_{s}, \lambda_{v}, \lambda_{0}, u\right)$ the Hamiltonian of the system defined by:

$$
\begin{aligned}
H:=-\lambda_{s} \mu(s) & {\left[\frac{M}{v}-\left(s-s_{i n}\right)\right] } \\
& +u\left[\frac{\lambda_{s}\left(s_{i n}-s\right)}{v}+\lambda_{v}\right]+\lambda_{0} .
\end{aligned}
$$

If $u$ is an optimal control and $(s, v)$ the corresponding solution of (9), there exists $t_{f}>0, \lambda_{0} \leq 0$, and an absolutely continuous map $\lambda=\left(\lambda_{s}, \lambda_{v}\right):\left[0, t_{f}\right] \rightarrow \mathbb{R}^{2}$ such that $\left(\lambda_{0}, \lambda\right) \neq 0, \dot{\lambda}_{s}=-\frac{\partial H}{\partial s}, \dot{\lambda}_{v}=-\frac{\partial H}{\partial v}$, that is:

$$
\left\{\begin{array}{l}
\dot{\lambda}_{s}=\lambda_{s}\left(\mu^{\prime}(s) x-\mu(s)+\frac{u}{v}\right) \\
\dot{\lambda}_{v}=\lambda_{s}\left(\frac{-\mu(s) M+u\left(s_{i n}-s\right)}{v^{2}}\right)
\end{array}\right.
$$

and we have the maximality condition:

$$
u(t) \in \operatorname{argmax}_{\omega \in[0,1]} H\left(s(t), v(t), \lambda_{s}(t), \lambda_{v}(t), \lambda_{0}, \omega\right),
$$

for almost every $t \in\left[0, t_{f}\right]$. We call extremal trajectory a sextuplet $\left(s(\cdot), v(\cdot), \lambda_{s}(\cdot), \lambda_{v}(\cdot), \lambda_{0}, u(\cdot)\right)$ satisfying (9)(16)-(17), and extremal control the control $u$ associated to this extremal trajectory. As $t_{f}$ is free, the Hamiltonian is zero along an extremal trajectory. Following [1], one can prove that $\lambda_{s}$ is always non-zero (it is therefore of constant sign from the adjoint equation), and that $\lambda_{0}<0$ (hence we take $\lambda_{0}=-1$ in the following). Next, let us define the switching function $\phi$ associated to the control $u$ by:

$$
\phi:=\frac{\lambda_{s}\left(s_{i n}-s\right)}{v}+\lambda_{v} .
$$

We obtain from (17) that any extremal control satisfies the following control law: for a.e. $t \in\left[0, t_{f}\right]$, we have

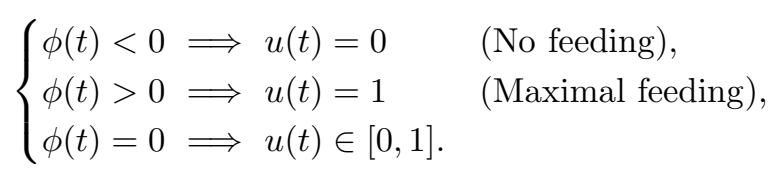

We say that $t_{0}$ is a switching point if the control $u$ is non-constant in any neighborhood of $t_{0}$ which implies that $\phi\left(t_{0}\right)=0$. In this case, the control is bang-bang around $t_{0}$, that is $u$ switches either from 0 to 1 or from 1 to 0 at time $t_{0}$. Whenever $\phi$ is zero on a non-trivial interval $I \subset\left[0, t_{f}\right]$, we say that $u$ is a singular control, and that the trajectory contains a singular arc. The sign of $\dot{\phi}$ is fundamental in order to obtain the optimal synthesis. By taking the derivative of $\phi$, we get:

$$
\dot{\phi}=\frac{\lambda_{s} x\left(s_{i n}-s\right) \mu^{\prime}(s)}{v} .
$$

Moreover, we can show that $\lambda_{s}<0$ (see e.g. [7], [1]). This implies that any extremal trajectory satisfies the property:

$$
s(t)>\bar{s} \Longrightarrow \dot{\phi}(t)>0 ; s(t)<\bar{s} \Longrightarrow \dot{\phi}(t)<0 .
$$

Now, if an extremal trajectory contains a singular arc on some time interval $I:=\left[t_{1}, t_{2}\right]$, then we have $\phi=\dot{\phi}=0$ on $I$, hence we have $\mu^{\prime}(s)=0$ and $s=\bar{s}$ on $I$. Therefore, by solving $\dot{s}=0$, we obtain easily the expression of the singular control given by (14), see e.g. [2].

Moreover, we can estimate the time of a singular trajectory as follows (see e.g. [1]):

$$
t_{2}-t_{1}=\frac{1}{\mu(\bar{s})} \ln \left(\frac{M+v\left(t_{2}\right)\left[s_{i n}-\bar{s}\right]}{M+v\left(t_{1}\right)\left[s_{i n}-\bar{s}\right]}\right),
$$

\section{OPTIMAL SYNTHESIS WITHOUT CONTROLLABILITY ASSUMPTION}

In this part, we provide a description of optimal trajectories for problem (6) when (12) is not satisfied. To this end, we introduce solutions of the system backward in time that will allow us to describe where optimal trajectories have a switching point.

\section{A. Study of the domain $\mathcal{D}$}

In view of (12), we introduce a mapping $\eta:\left(0, s_{i n}\right) \rightarrow \mathbb{R}$ by

$$
\eta(s):=\frac{1}{\mu(s)}-\frac{M}{s_{i n}-s} .
$$

By definition of $\eta$, we have:

$$
u_{s}(v)=1 \Longleftrightarrow v=\eta(s)
$$

Now, we set $v^{*}:=\eta(\bar{s})$ so that the singular arc is controllable provided that $v^{*} \geq v_{m}$. Therefore, we assume throughout this section that $v^{*}$ satisfies:

$$
v^{*}<v_{m}
$$

It follows that the singular arc is controllable only provided that the volume is less than $v^{*}$. Indeed, for $v>v^{*}$ equality (14) no longer defines an admissible control in $[0,1]$. Next, we will consider the two following cases: 
- Case 1: $v^{*} \leq 0$,

- Case 2: $0<v^{*}<v_{m}$.

Remark 1: Case 1 means that the singular arc is never controllable over $\left(0, v_{m}\right]$. As the function $\eta$ can take negative values, $v^{*}$ can be negative.

We now define the curve $\hat{\mathcal{C}}$ (resp. $\check{\mathcal{C}}$ ) whose graph is the mapping $v \in\left(0, v_{m}\right] \longmapsto \hat{\gamma}(v)$ (resp. $\left.v \in\left(0, v_{m}\right] \longmapsto \check{\gamma}(v)\right)$ as follows. The mapping $\hat{\gamma}$ (resp. $\check{\gamma}$ ) is the unique solution of the equation:

$$
\frac{d s}{d v}=-\mu(s)\left[\frac{M}{v}+s_{i n}-s\right]+\frac{s_{i n}-s}{v},
$$

which passes through the point $\left(\bar{s}, v_{m}\right)$ (resp. $\left.\left(\bar{s}, v^{*}\right)\right)$. In other words, $\hat{\gamma}(v)$ and $\check{\gamma}(v)$ are solution of (9) backward in time with a constant control $u=1$. These curves will play a major role in our optimal synthesis contrary to the case where the singular arc is controllable (see Figure 2 and Table I for parameter values). In fact, they will indicate sub-domains where optimal trajectories have a switching point.

Let $\hat{v}$ (resp. $\check{v}$ ) the first volume value such that $\hat{\gamma}(\hat{v}) \notin$ $\left(0, s_{i n}\right)$ (resp. $\left.\check{\gamma}(\check{v}) \notin\left(0, s_{i n}\right)\right)$. The next proposition is concerned with monotonicity properties of $\hat{\gamma}$ and $\check{\gamma}$.

Proposition 1: (i) The curve $\hat{\gamma}$ is either decreasing on $\left[\hat{v}, v_{m}\right]$, either there exists a unique $v_{1} \in\left(\hat{v}, v_{m}\right)$ such that $\hat{\gamma}\left(v_{1}\right) \in\left(0, s_{i n}\right)$ and $\frac{d \hat{\gamma}}{d v}\left(v_{1}\right)=0$. Moreover, in the latter case, $\hat{\gamma}$ is increasing on $\left[\hat{v}, v_{1}\right]$ and is decreasing on $\left[v_{1}, v_{m}\right]$. (ii) The mapping $\check{\gamma}$ is increasing on $\left(\check{v}, v^{*}\right]$ and decreasing on $\left[v^{*}, v_{m}\right]$ and $\frac{d \hat{\gamma}}{d v}\left(v^{*}\right)=0$.

Proof: We omit the proof that can be found in [3].

Remark 2: The previous proposition implies that the backward curve $\breve{\mathcal{C}}$ leaves the domain $\mathcal{D}$ through the linesegment $\{0\} \times\left[0, s_{\text {in }}\right]$ whereas $\hat{\mathcal{C}}$ leaves the domain $\mathcal{D}$ either through the line-segment $\{0\} \times\left[0, s_{i n}\right]$ or through the linesegment $\left\{s_{i n}\right\} \times\left[0, s_{i n}\right]$, see Figure 1 .

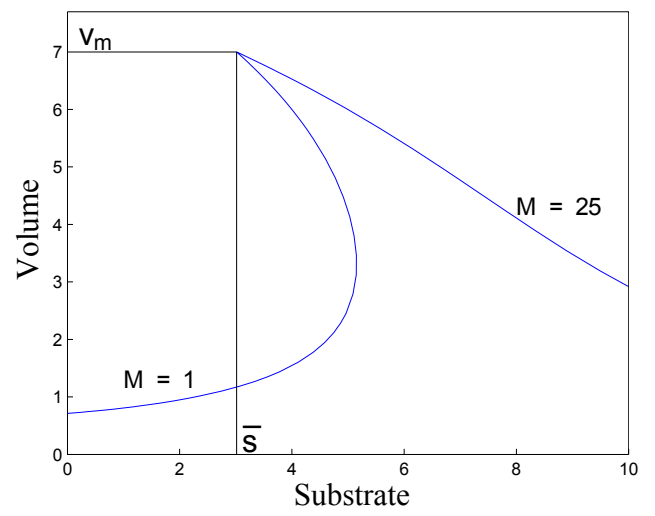

Fig. 1. The curve $\hat{\mathcal{C}}$ leaves the domain through $\{0\} \times\left[0, s_{i n}\right]$ when $M=1$, and through $\left\{s_{i n}\right\} \times\left[0, s_{i n}\right]$ when $M=25$.

In the second case of Proposition 1 (i), we can show that there exists values of $M$ such that there exists a unique $v_{*} \in$ $\left(0, v^{*}\right)$ such that:

$$
\hat{\gamma}\left(v_{*}\right)=\bar{s}
$$

see Fig. 2.

B. Optimal synthesis when the singular arc is never admissible

In the case where $v^{*} \leq 0$ (case 1 ), we have the following optimality result (see also Fig. 2).

Proposition 2: Assume that $v^{*} \leq 0$. Then, optimal controls satisfy the following:

- If $s_{0} \leq \bar{s}$, then, there exists $t_{0}>0$ such that $u=1$ on $\left[0, t_{0}\right], u=0$ on $\left[t_{0}, t_{f}\right]$ where $t_{0}$ is such that $v\left(t_{0}\right)=$ $v_{m}$ and $s\left(t_{f}\right)=s_{\text {ref }}$.

- If $\bar{s}<s_{0}<\hat{\gamma}\left(v_{0}\right)$, then, there exists $t_{0}>0$ such that $u=0$ on $\left[0, t_{0}\right], u=1$ on $\left[t_{0}, t_{1}\right], u=0$ on $\left[t_{1}, t_{f}\right]$ where $t_{0} \geq 0, \bar{s}<s\left(t_{0}\right)<s_{0}, v\left(t_{1}\right)=v_{m}$, and $s\left(t_{f}\right)=s_{\text {ref }}$.

- If $s_{0} \geq \hat{\gamma}\left(v_{0}\right)$, then, there exists $t_{0}>0$ such that $u=0$ on $\left[0, t_{0}\right], u=1$ on $\left[t_{0}, t_{1}\right], u=0$ on $\left[t_{1}, t_{f}\right]$ with $t_{0}>$ $0, \bar{s}<s\left(t_{0}\right)<\hat{\gamma}\left(v_{0}\right), v\left(t_{1}\right)=v_{m}$, and $s\left(t_{f}\right)=s_{\text {ref }}$.

Proof: We omit the proof that can be found in [3].

Remark 3: In the second case of proposition 2, the switching time $t_{0}$ from $u=0$ to $u=1$ may be zero and it can be found numerically, see section IV.

Next, we consider the second case where $0<v^{*}<v_{m}$. It is easy to see that for initial conditions such that $v_{0}>$ $v^{*}$, optimal controls are given by proposition 2 (indeed, the singular arc is defined only for $\left.v_{0} \leq v^{*}\right)$. Therefore, we only deal with $v_{0}<v^{*}$. The next Proposition is illustrated on Fig. 2.

Proposition 3: Assume that $v_{0}<v^{*}$ and that there exists $0<v_{*}<v^{*}$ such that $\hat{\gamma}\left(v_{*}\right)=\bar{s}$. Then, optimal controls satisfy the following:

- If $s_{0} \leq \check{\gamma}\left(v_{0}\right)$, then, there exists $t_{0}>0$ such that we have $u=1$ on $\left[0, t_{0}\right], u=0$ on $\left[t_{0}, t_{f}\right]$ where $t_{0}$ is such that $v\left(t_{0}\right)=v_{m}$.

- If $\check{\gamma}\left(v_{0}\right)<s_{0}<\hat{\gamma}\left(v_{0}\right)$ and $s_{0} \leq \bar{s}$, then, there exists $t_{0}>0$ such that we have $u=1$ on $\left[0, t_{0}\right], u=u_{s}$ on $\left[t_{0}, t_{1}\right], u=1$ on $\left[t_{1}, t_{2}\right], u=0$ on $\left[t_{2}, t_{f}\right]$, where $s\left(t_{0}\right)=\bar{s}, t_{1}-t_{0} \geq 0, v\left(t_{1}\right)<v^{*}$, and $v\left(t_{2}\right)=v_{m}$.

- If $\hat{\gamma}\left(v_{0}\right) \leq s_{0}<\bar{s}$, then, there exists $0<t_{0}<t_{1}<t_{2}$ such that we have $u=1$ on $\left[0, t_{0}\right], u=u_{s}$ on $\left[t_{0}, t_{1}\right]$, $u=1$ on $\left[t_{1}, t_{2}\right], u=0$ on $\left[t_{2}, t_{f}\right]$ where $s\left(t_{0}\right)=\bar{s}$, $v\left(t_{1}\right) \in\left(v_{*}, v^{*}\right), v\left(t_{2}\right)=v_{m}$.

- If $s_{0} \geq \bar{s}$ and $v_{0} \leq v_{*}$, then, there exists $0<t_{0}<$ $t_{1}<t_{2}$ such that we have $u=0$ on $\left[0, t_{0}\right], u=u_{s}$ on $\left[t_{0}, t_{1}\right], u=1$ on $\left[t_{1}, t_{2}\right], u=0$ on $\left[t_{2}, t_{f}\right]$ where $s\left(t_{0}\right)=\bar{s}, v\left(t_{1}\right) \in\left(v_{*}, v^{*}\right)$, and $v\left(t_{2}\right)=v_{m}$.

- If $s_{0} \geq \bar{s}$, and $v_{0}>v_{*}$, then, the optimal control is one of the following types:

- either $u=0$ on $\left[0, t_{0}\right], u=u_{s}$ on $\left[t_{0}, t_{1}\right], u=1$ on $\left[t_{1}, t_{2}\right], u=0$ on $\left[t_{2}, t_{f}\right]$ where $s\left(t_{0}\right)=\bar{s}$ and $0<t_{0}<t_{1}, v\left(t_{2}\right)=v_{m}$,

- either $u=0$ on $\left[0, t_{0}\right], u=1$ on $\left[t_{0}, t_{1}\right], u=0$ on $\left[t_{1}, t_{f}\right]$ where $t_{0} \geq 0, \bar{s}<s\left(t_{0}\right)<\hat{\gamma}\left(v_{0}\right), v\left(t_{1}\right)=$ $v_{m}$.

Proof: The proof that can be found in [3]. 
TABLE I

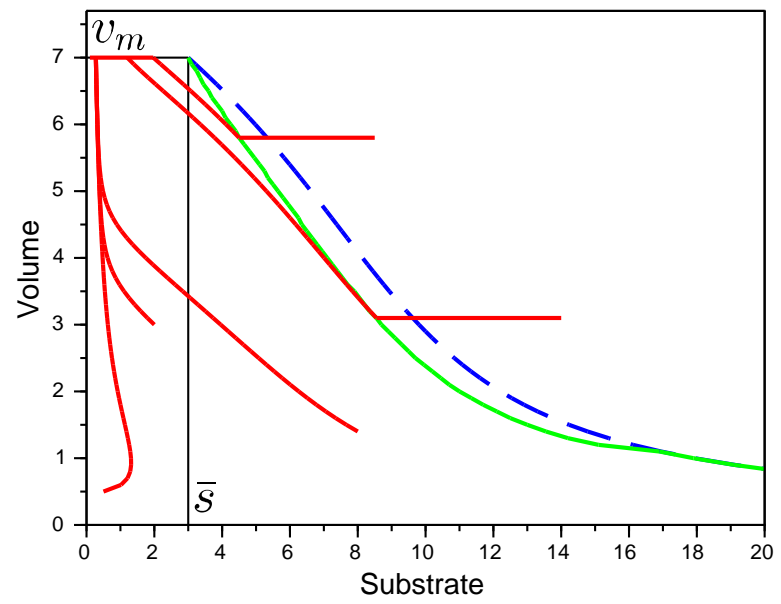

PARAMETER VALUES (ARBITRARY UNITS) OF SIMULATIONS FOR THE OPTIMAL SYNTHESIS (SEE FIG. 2 AND 3)

\begin{tabular}{ccccccc}
\hline$v_{m}$ & $s_{\text {in }}$ & $s_{\text {ref }}$ & $M$ & $\bar{\mu}$ & $k$ & $g$ \\
\hline 7 & 10 & 0.1 & $\begin{array}{c}25 \text { (case 1) } \\
1 \text { (case 2) }\end{array}$ & 0.5 & 1 & 0.11 \\
\hline
\end{tabular}

$t_{a}\left(v_{0}\right)$ of this strategy is (recall (20), see also [1]):

$$
\begin{aligned}
t_{a}\left(v_{0}\right)= & \frac{\ln \left(\frac{M+v_{0}\left(s_{i n}-\bar{s}\right)}{M+v_{*}\left(s_{i n}-\bar{s}\right)}\right)}{\mu(\bar{s})}+v_{m}-v_{0} \\
& +\int_{s_{\text {ref }}}^{s^{\dagger}\left(v_{0}\right)} \frac{d \sigma}{\mu(\sigma)\left(\frac{M}{v_{m}}+s_{i n}-\sigma\right)},
\end{aligned}
$$

where $s^{\dagger}(v)$ is the substrate concentration when the trajectory reaches $v=v_{m}$. We now show that $\tau$ admits a minimum $v_{a} \in\left[v_{*}, v^{*}\right]$ that we will characterize hereafter. First, notice that $s^{\dagger}\left(v_{0}\right)$ is obtained after integrating the ODE:

$$
\frac{d s}{d v}=-\mu(s)\left[\frac{M}{v}+s_{i n}-s\right]+\frac{s_{i n}-s}{v}, s\left(v_{0}\right)=\bar{s}
$$

Moreover, (26) can be equivalently written as $\frac{d s}{d v}=g(v, s)$ where $g$ is the right hand-side of (26). By the classical dependence of the solution of an ODE on parameters, we denote by $s\left(v, \bar{s}, v_{0}\right)$ the unique solution of (26). It is standard that $v_{0} \in \mathbb{R}_{+}^{*} \longmapsto s\left(v, \bar{s}, v_{0}\right)$ is of class $C^{1}$ for all $v>0$. It follows by composition that $v_{0} \longmapsto t_{a}\left(v_{0}\right)$ is of class $C^{1}$ on $\left[v_{*}, v^{*}\right]$. Consequently, it admits a minimum on this interval. By differentiating $s\left(v, \bar{s}, v_{0}\right)$ w.r.t. $v_{0}$, we get:

$$
\frac{\partial s}{\partial v_{0}}\left(v, \bar{s}, v_{0}\right)=-g\left(v_{0}, \bar{s}\right) e^{\int_{v_{0}}^{v} \frac{\partial g}{\partial s}\left(s\left(w, v_{0}, \bar{s}\right), w\right) d w},
$$

Fig. 2. Optimal trajectories (in solid red lines) for various initial conditions Top: case $1\left(v^{*} \leq 0\right)$, see Proposition 2 for the optimal synthesis; bottom: case $2\left(0<v^{*}<v_{m}\right)$, see Proposition 3. In blue dashed lines, trajectories $\hat{\mathcal{C}}$ and $\breve{\mathcal{C}}$ which pass through $\left(\bar{s}, v^{*}\right)$ and $\left(\bar{s}, v_{m}\right)$. In green, the mapping $v_{0} \longmapsto s_{b}\left(v_{0}\right)$ (see Section IV).

Remark 4: For the last item of the previous Proposition, the optimal trajectory is either $B_{0}-S A-B_{1}-B_{0}$ or $B_{0}-$ $B_{1}-B_{0}$ where $B_{0}$ (resp. $B_{1}$ ) denotes an arc Bang $u=0$ (resp. $u=1$ ), and $S A$ denotes a singular arc.

The next section will clarify this remark from a numerical point of view, and will provide an estimation of the switching time $t_{0}$ for items 2, 3, and 4 of Proposition 3.

\section{NUMERICAL SIMULATIONS}

\section{A. Determination of the maximal optimal volume}

We will focus on the second case $\left(0<v^{*}<v_{m}\right)$. Our aim in this part is to determine the optimal volume, denoted $v_{a}$, above which it is not optimal to stay on the singular arc, as follows.

For $v_{0} \in\left[v_{*}, v^{*}\right]$, consider the strategy $u=\bar{u}_{s}$ from $v_{*}$ to $v_{0}, u=1$ until $v_{m}$ and then $u=0$ until $s_{\text {ref }}$. The time

hence $\frac{d s^{\dagger}}{d v_{0}}\left(v_{0}\right)=\frac{\partial s}{\partial v_{0}}\left(v_{m}, \bar{s}, v_{0}\right)$. We have used a classical resul in ODE on the dependance of the trajectory w.r.t. initial conditions.

Now, we know from the PMP that $v_{0}=v^{*}$ and $v_{0}=$ $v_{*}$ are not admissible (see also Proposition 3), hence $v_{a}$ necessarily satisfies $\frac{d t_{a}}{d v_{0}}\left(v_{a}\right)=0$. So, if we put $\theta\left(v_{0}\right):=$ $\int_{v_{0}}^{v_{m}} \frac{\partial g}{\partial s}\left(w, s\left(w, \bar{s}, v_{0}\right)\right) d w$, we obtain by taking the derivative of (25) w.r.t. $v_{0}$ :

$$
\frac{d t_{a}}{d v_{0}}\left(v_{0}\right)=\frac{v^{*}-v_{0}}{\frac{M}{s_{i n}-\bar{s}}+v_{0}}\left[1-\frac{\mu(\bar{s})\left(\frac{M}{v_{0}}+s_{i n}-\bar{s}\right)}{\mu\left(s^{\dagger}\right)\left(\frac{M}{v_{m}}+s_{i n}-\bar{s}\right)} e^{\theta\left(v_{0}\right)}\right]
$$

This equation allows to obtain numerically the volume $v_{a} \in\left(v_{*}, v^{*}\right)$ above which extremal trajectories stop to be singular. As an example, we find $v_{a} \simeq 1.67$ (see Fig. 3 and Table I for the values of the parameters).

\section{B. Determination of the switching curve}

Now, we determine the optimal switching time for the trajectories $B_{0} B_{1} B_{0}$ starting with $s_{0}>\bar{s}$.

For each $v_{0} \in\left(v_{*}, v_{m}\right)$, we search numerically $s_{b}\left(v_{0}\right) \in$ $\left[\bar{s}, \hat{\gamma}\left(v_{0}\right)\right]$ which minimizes the time $t_{b}\left(s_{b}\right)$ to reach the target 
starting from $\left(\hat{\gamma}\left(v_{0}\right), v_{0}\right)$ with the strategy: $u=0$ until $s_{b}$, $u=1$ until $v_{m}, u=0$ until $s_{\text {ref }}$. Assuming that for each $v_{0}$ there is a unique minimum, this allows us to define the curve $\mathcal{C}_{b}$ whose graph is the mapping $v_{0} \longmapsto s_{b}\left(v_{0}\right)$, represented in green on Fig. 2. We find that for $v_{0}<v_{a}$, we have $s_{b}\left(v_{0}\right)=$ $\bar{s}$, while $s_{b}\left(v_{0}\right)>\bar{s}$ for $v_{0} \in\left(v_{a}, v_{m}\right)$. This result allows to conclude numerically which structure is optimal whenever $s_{0}>\bar{s}$ and $v_{0}>v_{*}$ (see the last case of Proposition 3 ):

- if $v_{0} \leq v_{a}$, the optimal strategy is $u=0$ on $\left[0, t_{0}\right]$, $u=u_{s}$ on $\left[t_{0}, t_{1}\right], u=1$ on $\left[t_{1}, t_{2}\right], u=0$ on $\left[t_{2}, t_{f}\right]$ where $s\left(t_{0}\right)=\bar{s}, v\left(t_{1}\right)=v_{a}, v\left(t_{2}\right)=v_{m}$.

- if $v_{0}>v_{a}$, the optimal strategy is $u=0$ on $\left[0, t_{0}\right]$, $u=1$ on $\left[t_{0}, t_{1}\right], u=0$ on $\left[t_{1}, t_{f}\right]$ where $t_{0} \geq 0$, $s\left(t_{0}\right)=\min \left(s(0), s_{b}\left(v_{0}\right)\right), v\left(t_{1}\right)=v_{m}$.

To conclude, based on the optimal synthesis (Proposition 3 ) and the computations of the mapping $v_{0} \longmapsto s_{b}\left(v_{0}\right)$ and $v_{a}$, optimal trajectories for various initial conditions $\left(s_{0}, v_{0}\right)$ are represented on Fig. 2. We have shown that the optimal synthesis is quite different as the one of Theorem 2. In particular, we have pointed out that it is not optimal for a trajectory to stay as long as possible on the singular arc. Moreover, the curve $v_{0} \longmapsto s_{b}\left(v_{0}\right)$ is the switching curve for optimal trajectories and it will allow to give an optimal feedback control.

We thus obtain the following optimal feedback control.

Proposition 4: The optimal feedback steering any initial state in $\mathcal{D}$ to the target is given by:

$$
u_{2}[s, v]:=\left\{\begin{array}{l}
0 \text { if } v=v_{m} \\
0 \text { if } s>s_{b}(v), \quad v \geq v_{a} \\
1 \text { if } s \leq s_{b}(v), \quad v_{m}>v \geq v_{a} \\
0 \text { if } s>\bar{s}, \quad v<v_{a} \\
1 \text { if } s<\bar{s}, \quad v<v_{a} \\
u_{s}(v) \text { if } s=\bar{s}, \quad v<v_{a},
\end{array}\right.
$$

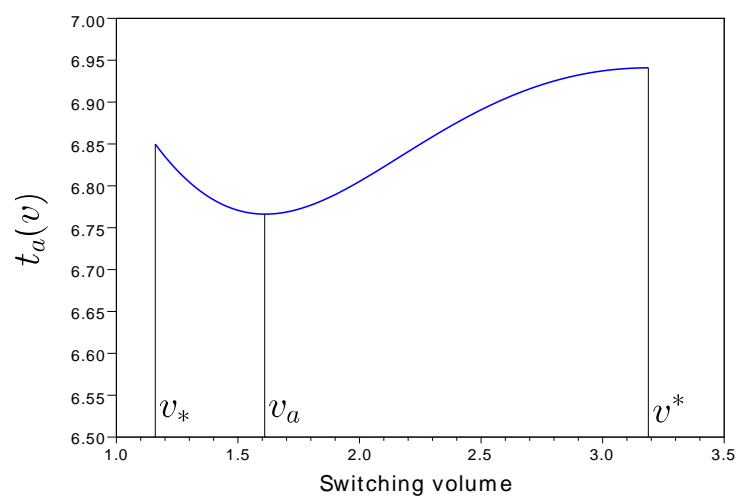

Fig. 3. Time $t_{a}(v)$ to reach $\mathcal{T}$ from $\left(\bar{s}, v_{*}\right)$ with the strategy: singular arc until the switching volume $v, u=1$ until $v_{m}, u=0$ until $s_{\text {ref }}$. We find that $t_{a}(v)$ has a unique minimum for $v=v_{a}$ (see Section IV)

\section{CONCLUSION}

We have studied an optimal control problem for a fedbatch bioprocess in presence of a non-controllable singular arc. This situation can arise for example when the initial biomass concentration is high. Thanks to Pontryagin Maximum Principle and a careful study of the switching function, we have obtained a characterization of optimal trajectories of the problem. Our main result is that it is not optimal for a trajectory to stay as long as possible on the singular arc. Moreover, we have obtained switching curves that can be used to determine an optimal feedback control of the problem. A more detailed insight into the determination of these switching curves (for instance using the theory of conjugate points [4]) will deserve more investigation. The analysis of the problem when the singular arc is nonadmissible everywhere is more delicate as in the standard case. We believe that this kind of study can be also the starting point to study optimal control problems in a more general setting in presence of non-controllable singular arcs.

The implementation of the optimal strategy will also deserve future works. Given that model parameters are generally poorly known, and that all the state variables cannot be measured, it will be a key challenge to determine on-line the switching points.

\section{ACKNOWLEDGEMENTS}

We would like to thank Professor C. Lobry and A. Rapaport for helpful discussions on the subject.

\section{REFERENCES}

[1] T. Bayen, P. Gajardo, F. Mairet, Optimal synthesis for the minimal time control problems of fed-batch processes for growth functions with two maxima, J. Optim. Theory and Applications, vol. 158, 2, pp 521-553, 2013.

[2] T. BAYEn, F. MAIRET, Minimal time control of fed-batch bioreactor with product inhibition Bioprocess and Biosystems Engineering, vol. 36, 10, pp. 1485-1496, 2013.

[3] T. BAyen, F. MAiret, M. Mazade, Minimal time problem for a fed-batch bioreactor with saturating singular control, preprint 2013, http://hal.archives-ouvertes.fr/hal-00879385.

[4] B. Bonnard, J.-B. Caillau, E. TrÉlat, Second order optimality conditions in the smooth case and applications in optimal control, ESAIM Control Optim. Calc. Var. 13, 2, pp. 207-236, 2007.

[5] B. Bonnard, M. Chy BA, Singular Trajectories and their role in Control Theory, Springer, SMAI, vol. 40, 2002.

[6] U. Boscain, B. PICCOLI, Optimal Syntheses for Control Systems on 2-D Manifolds, Springer SMAI, vol. 43, (2004)/

[7] D. Dochain, A. RAPAPORT, Minimal time control of fed-batch processes for growth functions with several maxima, IEEE Transactions on Automatic Control, vol. 56, 11, pp. 2671-2676, 2011.

[8] P. Gajardo, H. Ramirez, A. Rapaport, Minimal time sequential batch reactors with bounded and impulse controls for one or more species, SIAM J. Control Optim., Vol. 47, 6, pp. 2827-2856, 2008.

[9] J. Lee, S.Y. Lee, S. Park, A.P.J. Middelberg, Control of fedbatch fermentations, Biotechnology advances, Vol. 17, 1, pp. 29-48, 1999.

[10] A. Miele, Application of Green's Theorem to the extremization of linear integrals. Symp. on Vehicle Systems Optimization, Garden City, New York, 1961.

[11] J. A. Moreno, Optimal time control of bioreactors for the wastewater treatment, Optim. Control Appl. Meth., 20, pp. 145-164, 1999.

[12] L. Pontryagin, V. Boltyanski, R. Gamkrelidze, E. Michtchenko, The Mathematical Theory of Optimal Processes, Wiley Interscience, New York, 1962.

[13] H.L. Smith, P. Waltman, The theory of the chemostat, Dynamics of microbial competition, Cambridge University Press, 1995. 